Progress on common aspects of the EU-supplied ITER diagnostics and prediction of diagnostic performance

L. C. Ingesson, D. J. Campbell, M. Cecconello, E. Ciattaglia, P. Dirken, N. Hawkes, M. Walsh, M. von Hellermann, S. Kálvin, P. McCarthy, O. Neubauer, and L. Petrizzi

Citation: Review of Scientific Instruments 77, 10F502 (2006);

View online: https://doi.org/10.1063/1.2217856

View Table of Contents: http://aip.scitation.org/toc/rsi/77/10

Published by the American Institute of Physics

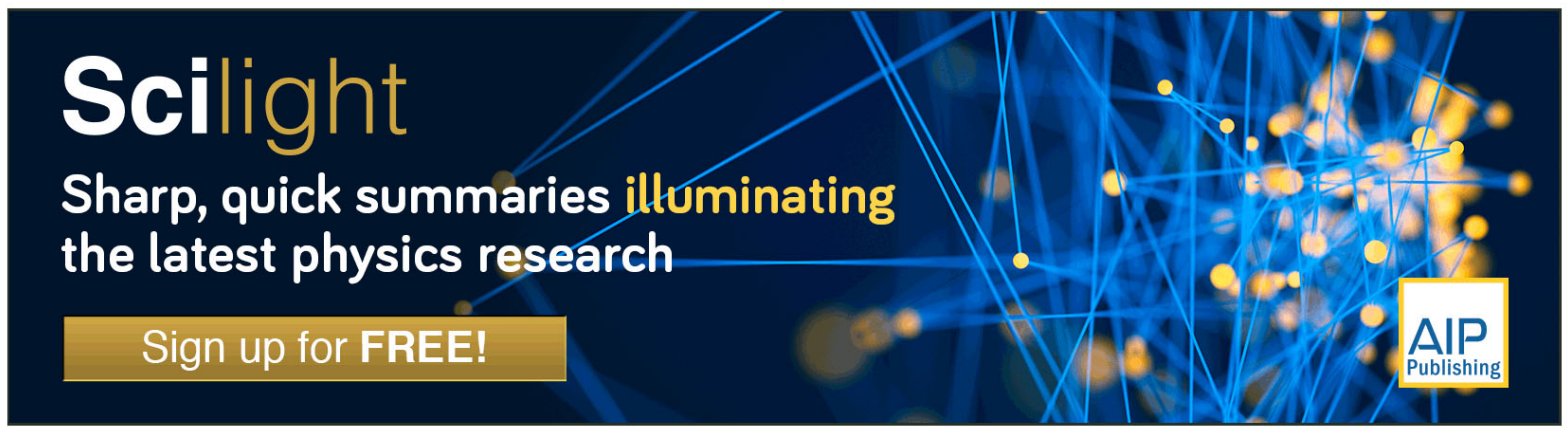




\title{
Progress on common aspects of the EU-supplied ITER diagnostics and prediction of diagnostic performance
}

\author{
L. C. Ingesson, ${ }^{\text {a) }}$ D. J. Campbell, M. Cecconello, ${ }^{\text {b) }}$ and E. Ciattaglia ${ }^{\text {() }}$ \\ EFDA Close Support Unit-Garching, Boltzmannstrasse 2, D-85748 Garching, Germany \\ P. Dirken, N. Hawkes, and M. Walsh \\ EURATOM-UKAEA Fusion Association, Culham Science Centre, OX14 3DB Abingdon, \\ Oxfordshire, United Kingdom \\ M. von Hellermann \\ FOM-Instituut voor Plasmafysica, Associatie Euratom-FOM, P.O. Box 1207, 3430 BE Nieuwegein, \\ The Netherlands \\ S. Kálvin \\ KFKI-Research Institute for Particle and Nuclear Physics, Association Euratom-HAS, P.O. Box 49, \\ Budapest-114, H-1525, Hungary \\ P. McCarthy \\ Department of Physics, University College Cork, Association Euratom-DCU, Cork, Ireland \\ O. Neubauer \\ IPP, Forschungszentrum Jülich GmbH, Association Euratom-FZJ, D-52425 Jülich, Germany \\ L. Petrizzi \\ Associazione ENEA-EURATOM sulla Fusione Frascati, Via E. Fermi, 45, I-00044 Frascati Rome, Italy
}

(Received 5 May 2006; presented on 11 May 2006; accepted 22 May 2006; published online 21 September 2006)

The European Union will supply a number of diagnostics for ITER. Significant progress has been made on the design and integration of these diagnostics. Specific topics, in common for several of the diagnostics, discussed are port-plug integration, simplification of labyrinths against neutron streaming, and design measures to protect and to be able to replace sensitive optical components. Performance analysis to predict the likely capability of the diagnostics to meet the ITER measurement requirements will be an important aspect of the design process. The interpretation of the measurement requirements, specifically of spatial resolution in the case of indirect measurements, is discussed on the basis of two examples, and methods of performance analysis are compared. () 2006 American Institute of Physics. [DOI: 10.1063/1.2217856]

\section{INTRODUCTION}

The European Union (EU) will supply a number of diagnostics for ITER, which are likely to include the plasmaposition reflectometer, the core-plasma charge-exchange recombination spectroscopy (CXRS) diagnostic, ${ }^{1}$ the LIDAR Thomson scattering diagnostic, ${ }^{2}$ the radial neutron camera, the visible/IR cameras on equatorial ports, the magnetics diagnostic, bolometers, pressure gauges, and thermocouples. The port plugs associated with the procurement packages that include these diagnostics will also be supplied.

Significant progress has been made on design and integration of these diagnostics, on $R \& D$ on critical components such as mirrors, and on development and testing of radiation

\footnotetext{
a) Also at FOM-Instituut voor Plasmafysica, Associatie Euratom-FOM, P. O. Box 1207, 3430 BE Nieuwegein, The Netherlands; electronic mail: christian.ingesson@efda.org

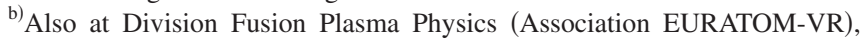
School of Electrical Engineering, Royal Institute of Technology KTH, S-10044 Stockholm, Sweden.

${ }^{c)}$ Also at EURATOM-UKAEA Fusion Association, Culham Science Centre, OX14 3DB Abingdon, Oxfordshire, United Kingdom.
}

hard components. This article will discuss a number of specific technical analyses and design solutions that several of the diagnostics have in common, and review methods of performance analysis as part of the design process.

\section{THE ITER ENVIRONMENT AND DIAGNOSTIC DESIGN CHALLENGES}

The ITER environment ${ }^{3}$ poses many challenges compared with the integration environment encountered on present fusion plasma devices. The radiation environment, with significant neutron and gamma fluxes in locations where diagnostics are to be integrated, causes volume heating in all components and places restrictions on the materials that can be used. For example, materials and components must be simultaneously vacuum and nuclear compatible, one must be able to cope with the changes in structural and physical properties, transmutation products must be harmless, and metal connections must be reweldable after activation. There are specific demands on optical diagnostics. Mirror labyrinths are used for mainly two reasons: the labyrinth reduces neutron streaming and reflective optics is more radiation hard than refractive optics. However, particle fluxes 
from the plasma onto the mirrors pose a severe challenge. Finally, the ITER environment dictates that the access for repair and maintenance will be very restricted, and thus diagnostic components must be designed with a very high degree of robustness and reliability. For many reasons, the portbased diagnostics are mounted in port plugs. Technical analysis and design solutions in common for several or the ITER diagnostics are discussed: port-plug integration, simplification of labyrinths against neutron streaming, and design measures to protect and to be able to replace sensitive optical components.

Design issues of upper and equatorial port plugs and integration issues in the divertor port are being addressed in a cross-party activity. The EU contributes to many of these. Manufacturing options for the port-plug structure have been assessed; forging of the components and gun-drilling cooling channels appear to be suitable manufacturing methods. Thermohydraulic analysis and hydraulic design (e.g., draining and drying analyses) are carried out in order to optimize the cooling channels, and a remote-handling orbital welded water connection is being designed.

The allowable neutron streaming through ITER ports is limited by the activation at the port flange (to allow manned intervention) and by the acceptable damage levels to materials (such as the superconducting magnets). Furthermore, the radiation level affects diagnostic materials and components. Neutronics analysis is therefore used as a design tool for the integration of diagnostic components. For example, a study on the far-forward mirrors of the CXRS system has revealed that the heating rates, and neutron and gamma fluxes can be reduced by as much as $70 \%-80 \%$ by recessing the first two mirrors by just $0.3 \mathrm{~m}$. For remote-handling installation and removal, a gap is needed between the port extension and the port plug. Even with measures to minimize neutron streaming through the gap, for most port plugs the gap is the dominant source of streaming. This provides a guide to the extent efforts are needed to limit streaming through diagnostic apertures and labyrinths. For the LIDAR diagnostic, changes from the three-mirror reference design are considered, such as an option with two mirrors for combined laser and scattered light and a two-mirror option with a direct laser beam. ${ }^{2}$ These options improve the diagnostic performance at the expense of a slightly higher neutron streaming. Similarly, an optical layout for the CXRS diagnostic with only one dogleg is being analyzed. ${ }^{1}$ A parametric study has been performed to quantify the neutron streaming through diagnostic labyrinths as a function of aperture size and dog-leg offset. A complicated nonlinear dependence on the two parameters is found, related to a threshold in neutron streaming when the offset is small compared with the aperture size. A straightforward rule of thumb for the labyrinth parameters to be used diagnostic design could therefore not be derived.

Extraction of a port plug for maintenance or replacement of components is a major undertaking, which, for each individual port plug, is likely to be only possible a few times over the ITER operating life. Sensitive optical components, such as mirrors, may need to be replaced more often. Optical components mounted on extractable tubes are therefore explored (Fig. 1). Such tubes still require a detailed engineering

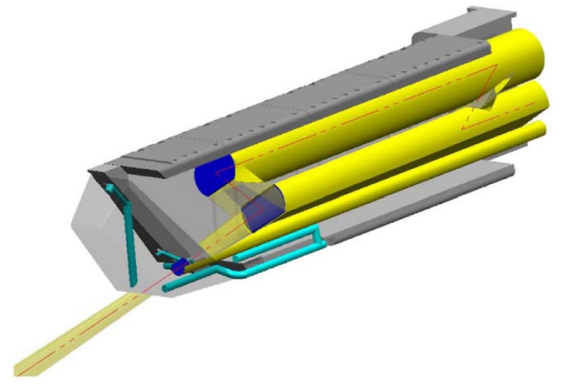

FIG. 1. (Color online) View of a port plug with one of the concepts of mirrors and shutter mounted on several extractable tubes.

feasibility study, including aspects such as flange attachment, steps in diameter to reduce neutron streaming, free-hanging or supported front, and insertion and handling mechanisms. Diagnostic shutter concepts are also being developed to protect the mirrors when not in use, requiring very reliable radiation-hard vacuum compatible components, transmitters of movement, and activators.

\section{PERFORMANCE ANALYSIS}

The ITER diagnostics will mainly be procured on the basis of functional specifications. Performance analysis to predict the likely capability of the diagnostics to meet the ITER measurement requirements is therefore an important aspect of the design process. The discussion on performance analysis here will focus on the cases in which the derivation of physical plasma parameters from the measurements involves an inverse mathematical operation. In addition to the two examples given, bolometers and $q$-profile (safety factor) determination, diagnostics that face similar issues, in particular on the interpretation of spatial resolution, include the $\mathrm{x}$-ray crystal spectroscopy imaging system, ${ }^{4}$ the magnetics diagnostic (reconstruction of magnetic equilibria), the radial neutron camera, the soft x-ray diagnostic (tomography), and the LIDAR diagnostics (deconvolution of laser pulse shape).

Performance analysis is generally carried out by (1) simulating diagnostic measurements for certain ITERrelevant plasma parameters, (2) reconstructing the relevant parameters (such as profiles) from those measurements, and (3) comparing the results with the input data. The performance analysis can either look at a small range of the relevant plasma parameters specifically related to the standard ITER scenarios, or by statistical means consider a large range of the relevant plasma parameters. In both cases the inverse problem can be solved by adequate mathematical tools. In the second case, the statistical performance analysis can also be performed by fitting techniques to a database of cases generated, by techniques such as function parametrization (FP) and neural networks.

Figure 2 shows an assessment of $q$-profile reconstruction capability by function parametrization on a database of a wide class of ITER equilibria from simulated magnetics, poloidal polarimeter, and motional Stark-effect (MSE) measurements in ITER. Iota-bar $\bar{\iota}=1 / q$ is used instead of $q$ because the error propagation is more well-behaved (homoscedastic). The range of equilibria was selected mainly on the basis of the technical capability of ITER rather than 


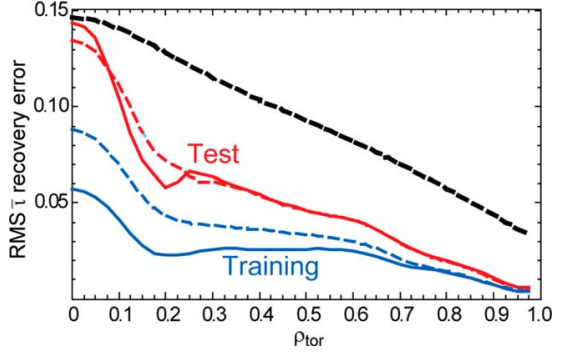

FIG. 2. (Color online) The best root-mean-squared recovery errors of $\bar{\iota}$ as a function of radius $\left(\rho_{\text {tor }}\right)$ from FP regression using magnetics data $[22$ principal components (PCs)], 16 polarimeter PCs and 12 MSE PCs (solid curves), and magnetics and MSE data only (thin dashed curves). The thick dashed curve indicates the $10 \%$ target accuracy of the RMS $\bar{\imath}$ in the database (calculated separately for each radius). The curves marked "training" show the training dataset results. The curves marked "test" show the recovery errors for the test dataset which was not included in the training stage.

being variations on the ITER standard scenarios. The figure shows that the target accuracy is met for the entire radius. The apparent lack of improvement when polarimetric data is added to MSE (solid versus dashed curves marked "test") may be due to shortcomings in the analysis method (FP model size).

To study the capability to derive accurate total emission profiles from the bolometer diagnostic and to optimize the geometry of lines of sight for this purpose, the type of analysis (tomographic reconstructions and many different conditions, such as noise levels and line-of-sight distributions) dictates that this can only be done for a limited range of different plasma conditions (such as a limited range of bulk plasma parameters and attached and detached conditions in the divertor). From a qualitative comparison of twodimensional emission profiles, one can infer the typical reconstruction-quality dependence on, for example, noise level (strong dependence) and number of lines of sight (weak dependence). Quantitative assessment of reconstruction quality is difficult for two-dimensional profile data. Derived quantities in one dimension (e.g., flux-surface averaged emissivity and cross sections) and in zero dimensions (e.g., total radiated power, radiated power within the separatrix, or radiated power in the divertor), however, can be shown graphically with error bars (from sensitivity analysis or statistical methods) and are a convenient and physically meaningful way to quantify the performance.

The definition of spatial resolution for profiles derived from nonlocal measurements, as is the case in many diagnostics, is not trivial and can be ambiguous. The example of a $q$-profile reconstruction shows that for reconstructed profiles the defined accuracy can be a sufficient requirement. The notion of spatial resolution, however, is useful to quantify spatial profile correlation lengths and gradients that should be resolvable. Possible definitions of spatial resolution can include information from statistical methods (e.g., number of meaningful nodes in spline fitting), the maximum grid size that does not affect the reconstructed profile, the smallest feature or gradient in the profile that can be distinguished, or the smallest distance between two features that can be resolved. These suggestions do not, or only partially, take into account measurement errors and the resulting effect on the reconstructed profiles. An alternative measure of spatial reso- lution [e.g., one dimensional in the radial direction] could be $\Delta \rho=\varepsilon(b) /(d b / d \rho)_{\mathrm{RMS}}$, where $b(\rho)$ is the radial profile quantity and $\varepsilon(b)$ the uncertainty in reconstructed profile. The root-mean-squared gradient over a database is taken to avoid mathematical problems with zero gradients in individual profiles.

The following conclusions can be inferred from a review of performance analysis methods. The choice of input assumptions (e.g., range of plasma parameters included) can have a large influence on the results. It may therefore be impossible to compare results with earlier analysis if the assumptions are significantly different. There may also be discrepancies because of differences between analysis codes or the specifics of the method. Hence, it is important to verify results with those from other analyses and benchmark codes using standardized input. The input assumptions should be considered carefully in line with the aim of the performance analysis. To obtain the most relevant information, it seems preferable to focus performance analysis on a narrow range of plasma parameters around the ITER standard scenarios. However, it is also relevant to verify the performance predictions for the much wider range of plasma parameters possible in ITER (e.g., as in the $q$-profile example). The choice between the statistical method (e.g., the $q$-profile example) or testing the performance on a few specific cases (e.g., the bolometry example) depends largely on what analysis is practical for a particular diagnostic. Interpretation of simulation results is far from trivial: it may depend on details of the analysis method used and it may be difficult to extract general conclusions from a large number of test cases.

\section{DISCUSSION}

Technical solutions are successfully being developed to overcome the significant challenges posed by diagnostic integration in ITER. Predicting the likely performance of diagnostics in the ITER environment is not trivial. Even if all technical details of the diagnostic and environment are known, the analysis method and input assumptions chosen may have a significant impact on the results and should be carefully considered to obtain meaningful predictions. The definition of a spatial-resolution target in the ITER measurement requirements is inadequate. Suggestions have been given for alternatives. An adequate choice will, however, depend on the analysis method (for example whether statistical information is available).

\section{ACKNOWLEDGMENTS}

This work, supported by the European Communities under various contracts of Association between EURATOM and EU Fusion Laboratories, was carried out within the framework of the European Fusion Development Agreement. The views and opinions expressed herein do not necessarily reflect those of the European Commission.

\footnotetext{
${ }^{1}$ M. von Hellermann et al., Rev. Sci. Instrum., these proceedings.

${ }^{2}$ M. J. Walsh et al., Rev. Sci. Instrum., these proceedings.

${ }^{3}$ A. E. Costley, D. J. Campbell, S. Kasai, K. E. Young, and V. Zaveriaev, Fusion Eng. Des. 55, 331 (2001).

${ }^{4}$ L. C. Ingesson, R. Barnsley, A. Malaquias and M. O'Mullane, Rev. Sci. Instrum. 75, 3696 (2004).
} 